\title{
FUZZY MULTIATTRIBUTE UTILITY ANALYSIS FOR COLLECTIVE CHOICE
}

Fumiko Seo

Kyoto Institue of Economic Research

Kyoto, Japan

Masatoshi Sakawa

Department of Systems Engineering

Kobe University, Japan

RR-87-13

July 1987

Reprinted from IEEE Transactions on Systems, Man, and Cybernetics, volume 15 (1985).

INTERNATIONAL INSTITUTE FOR APPLIED SYSTEMS ANALYSIS

Laxenburg, Austria 
Research Reports, which record research conducted at IIASA, are independently reviewed before publication. However, the views and opinions they express are not necessarily those of the Institute or the National Member Organizations that support it.

Reprinted with permission from IEEE Transactions on Sy'stems, Man, and Cybernetics 15(1):45-53. Copyright 두 1985, IEEE.

All rights reserved. No part of this publication may be reproduced or transmitted in any form or by any means, electronic or mechanical, including photocopy, recording, or any information storage or retrieval system, without permission in writing from the copyright holder.

Printed by Novographic, Vienna, Austria 


\section{FOREWORD}

Both authors of this paper are members of the System and Decison Sciences (SDS) core group at IIASA, and we are pleased to bring one example of their interesting work to the attention of IIASA Report readers.

In this paper the authors extend multiattribute utility analysis to the multiple agents decision problem. They are concerned in particular with fuzzy evaluation of the multiattribute utility function, which is based on a fuzzy preference ordering and scaling constants using membership functions of fuzzy set theory. Thus, their ideas are in the spirit of research currently being conducted at IIASA on the role of analysis in the policymaking process.

ALEXANDER B. KURZHANSKI

Leader

System and Decision Sciences 


\title{
Fuzzy Multiattribute Utility Analysis for Collective Choice
}

\author{
FUMIKO SEO AND MASATOSHI SAKAWA
}

\begin{abstract}
An extension of multiattribute utility analysis for the multiple-agents decision problem is presented. Although multiattribute utility analysis is concerned with decisionmaking under uncertainty, assessment of parameters of the multiattribute utility functions is actually performed deterministically by the single decisionmaker. This paper is concerned with fuzzy evaluation of the multiattribute utility function, which is based on a fuzzy preference ordering and scaling constants using membership functions of the fuzzy set theory. The fuzzy approach treats a conceptual imprecision that accrues from a multiplicity of evaluation. A fuzzy multiat tribute utility function with multiple-agents evaluation is derived. A computer package ICOPSS/FR for assuring the transitivity of collective preference ordering in an agreement level is demonstrated for assessment of the fuzzy multiattribute utility functions.
\end{abstract}

\section{INTRODUCTION}

$\mathbf{M}$ ULTIATTRIBUTE utility analysis has been developed for making decisions in the subjective phase of multiobjective evaluation and has shown to be particularly useful for manipulating noncommensurateness of the multidimensional and conflicting objectives. However, this method has neglected multiple-agents decision problems. Evaluation is exclusively based on individual preferences of the single decisionmaker. The method ultimately has some individual assert a set of his preferences as "socially" desirable. Collective choice or group decision problems are not taken into consideration. In addition, complete information is presumed in the assessment processes. Incompleteness or ambiguity of knowledge and information is not considered in heuristic processes of the parameter estimation for multiattribute utility functions. Those limitations of the multiattribute utility analysis are partially connected with each other and can be jointly considered to some extent.

Difficulty of the collective-choice decision problem has been well known. Arrow ([1], [2]) established the impossibility theorem of deriving a social utility function based on individual weak preference orderings under the assumption of nondictatorship. He insisted on the order-preserved utility functions measured with ordinal scales. Afterwards preference aggregation rules have been developed in terms of risky multiattribute utility functions under uncertainty (Keeney and Kirkwood [10], Keeney [9]) as well as nonrisky measurable multiattribute value functions under cer-

Manuscript reviewed April 25, 1984; revised September 28. 1984.

F. Seo is with the Kyoto Institute of Economic Research. Kyoto University, Kyoto 606. Japan.

M. Sakawa is with the Department of Systems Engineering. Kobe University, Kobe 657, Japan. tainty (Dyer and Sarlin [7], etc.). Representation theorems analogous to those of the multiattribute utility function of the single-decisionmaker have been derived for both the group utility and value functions. However assessment of those functions is difficult from a practical point of view because interpersonal comparisons of preferences should be carried out in both the cases; for which any general standard of measurement can not be directly established. This paper presents a collective-choice extension of the multiattribute utility analysis for the single-decisionmaker, which avoids construction of the group multiattribute utility function with the aggregation rules for individual preferences.

The collective-choice decision problem has two aspects: one is to consider a diversification of evaluation, and the other is to reconcile the value-conflicts among multiple assessors. This paper is concerned with articulating an agreement level or an acceptable range of the multiattribute utility function, which is derived from the diversified evaluations.

There are two ways to this direction of research. First is the probabilistic approach; which treats the objects to be evaluated as uncertain quantities and assesses their probability distributions. As a modification of the probabilistic approach, an entropy model can be used for assessing the probabilities. In this method, a probability is regarded as an index showing a diversification of evaluation by anonymous multiple-decisionmakers or the "public." An entropy maximization problem can be solved with one specific factor, utility, along with another criterion. This method has been used to risk assessment for implementing alternative regional policies with conflicting objectives (Seo and Sakawa [14]). The entropy approach is primarily based on the prior assessment of events to occur, which is analytically calculated.

Second is the fuzzy approach, which is nonprobabilistic and is concerned with the linguistic ambiguity of some assertions based on the posterior assessment via subjective decisions. Concepts for fuzzy preference relations (Zadeh [17]) and fuzzy number operations (Dubois and Prade [5], [6]) can be partially applied in the process of assessing the multiattribute utility function. A collective choice problem based on the fuzzy set theory has been considered by Blin [3], and Bin and Whinston [4]. However they have not cared of constructing utility functions for the collective choice problems. Any device for measuring agreement levels 
or ranges in the collective choice has not been presented in terms of the utility functions.

This paper is concerned with deriving the fuzzy multiattribute utility function (FMUF) for collective choice based on the fuzzy extensions of the multiattribute utility function (MUF). In context of the collective choice decision problems, the linguistic ambiguity of an assertion on the utility valuation comes from the diversification of evaluation by multiple assessors. Thus construction of FMUF can be performed as a natural extension of the fuzzy concepts to the preference valuation. Although this approach does not treat the value trade-offs among the assessors directly, it will be able to make clear quantitatively the levels or ranges of the value-conflicts existing among the multiple assessors.

In Section II, an outline of fuzzification device of the multiattribute utility analysis is described. In the Section III, rationality of collective choice is discussed in terms of a fuzzy preference ordering and its decomposition for defuzzification. In Section IV, a derivation process of a nonfuzzy collective preference ordering is described under the alternative rules for the collective choice. A computer utilization for assuring the transitivity in this process is demonstrated. In Section V, derivation of the fuzzy multiattribute utility function as a fuzzy number is discussed. In Section VI, a summary and concluding remarks are presented.

\section{Fuzzy Multiattribute Utility Analysis}

In general, the multiattribute utility function is assessed with the following representation theorem (Keeney [8], Keeney and Raiffa [11]).

Theorem 1: Given an objects set of evaluation (systems' attributes) $X \triangleq\left\{X_{i}\right\}$ and their performance levels $x \triangleq$ $\left\{x_{i}\right\}, i=1, \cdots, m$, then, under the assumptions on preferential and utility independence, either

$$
U\left(x_{1}, \cdots, x_{m}\right)=\sum_{i=1}^{m} k_{i} u_{i}\left(x_{i}\right)
$$

if

$$
\sum_{i=1}^{m} k_{i}=1
$$

or

$$
1+K U\left(x_{1}, \cdots, x_{m}\right)=\prod_{i=1}^{m}\left(1+K k_{i} u_{i}\left(x_{i}\right)\right)
$$

if

$$
\sum_{i=1}^{m} k_{i} \neq 1
$$

where $U$ and $u_{i}$ are utility functions scaled zero to one. $K$ and $k_{i}$ are called scaling constants and $0<k_{i}<1, K>$ -1 . When $\sum_{i=1}^{m} k_{i} \neq 1, K$ is a nonzero solution to

$$
1+K=\prod_{i=1}^{m}\left(1+K k_{i}\right) .
$$

The unique solution $K$ takes the ranges of $0<K<\infty$ when $\sum_{i=1}^{m} k_{i}<1$, and $-1<K<0$ when $\sum_{i-1}^{m} k_{i}>1$. It has been proved that the $K$-value can be determined uniquely in any high-order equation (3).

The procedure of assessing the MUF is composed of three steps:

1) evaluating the unidimensional (single-attribute) utility functions (UNIF), $u_{i}, i=1, \cdots, m$;

2) assessing the scaling constants $k_{i}$ and $K$ on them; and

3) obtaining the representation form (1) or (2) of the MUF.

Step 1) corresponds to the decomposition of systems. Step 3 ) is a simple calculation process. This paper intends to fuzzify the value assessment in the step 2), the coordination process, which includes evaluations by the multiple decisionmakers as the mediator.

In step 2), fuzzification can be performed twofold: before and after the value trade-off experiment among the attributes, which is executed for deriving relative values of the scaling constant $k_{i}$. First, before the experiment, objects (attributes) $X_{i}, i=1, \cdots, m$, are compared with each other and a fuzzy preference ordering among the objects is found. With a defuzzification device, a nonfuzzy collective preference ordering is derived. A numerical ordering of the scaling constants $k_{i}$ is determined corresponding to the nonfuzzy preference ordering among $X_{i}, i=1, \cdots, m$. The relative values $k_{i} / k_{s}, i \neq s$, of the scaling constants are found with the trade-off experiment based on the numerical ordering of them. Second, after the value trade-off experiment, a numerical value of the fuzzy scaling constant $\tilde{k}_{s}$ for the utility function $u_{s}\left(x_{s}\right)$ of the most preferable attribute $x_{s}$ is assessed as a fuzzy number. Thus all values of the fuzzy scaling constants $\tilde{k}_{i}, i=1, \cdots, m$, can be numerically determined. In the following section, the fuzzification process is discussed.

\section{Rationality of the Collective Choice}

\section{A. Fuzzy Preference Ordering}

Now the first phase of the fuzzification process in step 2) can be discussed, where the preference ordering among attributes should be derived.

In general, rationality of human behavior is expressed in terms of consistency and coherence of preference ordering. Consistency means asymmetry and coherence means transitivity. Thus the rationality should assure the strong ordering (viz. asymmetry and transitivity). However, it is not easy to fulfill both the properties in actual processes of the decisionmaking. Thus we use the weak (complete) ordering with connectivity as a relaxed surrogate of asymmetry in place of the strong ordering. The concepts of fuzzy preference ordering are defined with the membership function $\mu_{R}\left(X_{i}, X_{j}\right)$ for all $X_{i} \in \tilde{X}_{i}$ and $X_{j} \in \tilde{X}_{j}$, where $\tilde{X}_{i}$ and $\tilde{X}_{j}$ are fuzzy sets of evaluation objects. The membership function $\mu_{R}\left(X_{i}, X_{j}\right)$ associates with each pair $\left(X_{i}, X_{j}\right) \in \tilde{X}_{i} \times \tilde{X}_{j}$ its "grade of membership" $\mu_{R}$, where $R$ is a fuzzy binary relation $(\prec, \sim)$ for preferences defined on ordered pairs 
of the objects (attributes). The number of $\mu_{R}\left(X_{i}, X_{j}\right)$ that takes a range of interval $[0,1]$ represents a strength of the preference relation $R$ between $X_{i}$ and $X_{j}$.

Definition 1 (Fuzzy Weak Ordering): Fuzzy weak ordering is defined with connectivity and transitivity as follows.

1) Connectivity:

$X_{i} \neq X_{j} \Rightarrow \mu_{R}\left(X_{i}, X_{j}\right)>0$ or $\mu_{R}\left(X_{j}, X_{i}\right)>0$.

2) Transitivity:

$$
\mu_{R}\left(X_{i}, X_{k}\right) \geqslant \bigvee_{x_{j}}\left(\mu_{R}\left(X_{i}, X_{j}\right) \wedge \mu_{R}\left(X_{j}, X_{k}\right)\right)
$$

where $\mu_{R} \vee \mu_{R}^{\prime}$ denotes $\max \left(\mu_{R}, \mu_{R}^{\prime}\right)$ and $\mu_{R} \wedge \mu_{R}^{\prime}$ denotes $\min \left(\mu_{R}, \mu_{R}^{\prime}\right)$. When the connectivity is replaced by

3) Asymmetry:

$$
\mu_{R}\left(X_{i}, X_{j}\right)>0 \Rightarrow \mu_{R}\left(X_{j}, X_{i}\right) \ngtr 0,
$$

the preference ordering is called strong ordering.

\section{B. A Hierarchical Structure of the Fuzzy Preference Orderings}

A hierarchical structure of the fuzzy preference ordering can be considered in resolution of the fuzzy binary relation $R$ into a union of several nonfuzzy sets. For a number $\alpha$ in $[0,1]$, an $\alpha$-level set $R_{\alpha}$ of a fuzzy relation $R$ is defined by

$$
R_{\alpha}=\left\{\left(X_{i}, X_{j}\right) \mid \mu_{R}\left(X_{i}, X_{j}\right) \geqslant \alpha\right\} .
$$

The $R_{\alpha}$ is a nonfuzzy set of $X_{i} \times X_{j}$ and those sets form a nested sequence of nonfuzzy relations with $\alpha_{i} \geqslant \alpha_{j} \Rightarrow R_{\alpha_{i}}$ $\subset R_{\alpha}$. The $\alpha$ is interpreted as an agreement level of the collective choice.

According to the Zadeh's proposition [17], any fuzzy relation $R$ defined on $X \in\left(\tilde{X}_{1} \times \cdots \times \tilde{X}_{m}\right)$ admits of the resolution

$$
R=\sum_{\alpha} \alpha R_{\alpha}, \quad 0<\alpha \leqslant 1
$$

where $\Sigma_{\alpha}$ stands for the union $U_{\alpha}$, and $\alpha R_{\alpha}$ denotes a nonfuzzy set defined by

$$
\mu_{\alpha R_{a}}\left(X_{i}, X_{j}\right)=\alpha \mu_{R_{a}}\left(X_{i}, X_{j}\right)
$$

or equivalently

$$
\mu_{\alpha R_{\alpha}}\left(X_{i}, X_{j}\right)= \begin{cases}\alpha, & \text { for }\left(X_{i}, X_{j}\right) \in R_{\alpha} \\ 0, & \text { otherwise. }\end{cases}
$$

For the resolution of a fuzzy preference relation $R$ into the nonfuzzy subsets $R_{\alpha}$, the relation matrix whose elements are composed of $\mu_{R}$ can be constructed. The collective preference ordering can be generated by a permutation mapping $\psi: X \rightarrow X$, where $X$ denotes an objects set defined for a preference ordering. This permutation operation can be executed for constructing a nonfuzzy collective ordering from the fuzzy set of the individual preference orderings among the objects.

The hierachical structure of the fuzzy preference ordering is constructed for deriving a compromised preference ordering from diversified evaluation in the multiple-agents decisionmaking. In the next section an algorithm for wonstructing the compromised preference ordering among multiple assessors is discussed under the alternative selection rules.

\section{Derivation of a Nonfuzzy Collective PREFERENCE ORDERING}

\section{A. Constructing Relation Matrices}

Derivation of the nonfuzzy collective preference ordering from a fuzzy set of individual preference orderings of multiple decisionmakers depends on selection rules for the collective choice.

1) Simple Majority Rule:

$$
\mu_{R}\left(X_{i}, X_{j}\right) \triangleq \frac{1}{n} N\left(0_{i j}\right)
$$

where $n$ is a number of assessors, and $N\left(0_{i j}\right)$ denotes a total score (e.g., number of votes) for the pairwise preference ordering $0_{i j}$ between the attributes $X_{i}$ and $X_{j}$. For instance, suppose that $n=20, X \triangleq\left(X_{1}, X_{2}, X_{3}, X_{4}\right)$ and a score sheet for the alternative quaternary assessment can be obtained as follows

$$
\begin{array}{ll}
0^{1}=\left(X_{1}, X_{2}, X_{3}, X_{4}\right), & N\left(0^{1}\right)=4 \\
0^{2}=\left(X_{1}, X_{2}, X_{4}, X_{3}\right), & N\left(0^{2}\right)=2 \\
0^{3}=\left(X_{2}, X_{1}, X_{3}, X_{4}\right), & N\left(0^{3}\right)=2 \\
0^{4}=\left(X_{2}, X_{1}, X_{4}, X_{3}\right), & N\left(0^{4}\right)=1 \\
0^{5}=\left(X_{3}, X_{1}, X_{2}, X_{4}\right), & N\left(0^{5}\right)=2 \\
0^{6}=\left(X_{3}, X_{1}, X_{4}, X_{2}\right), & N\left(0^{6}\right)=1 \\
0^{7}=\left(X_{1}, X_{3}, X_{2}, X_{4}\right), & N\left(0^{7}\right)=3 \\
0^{8}=\left(X_{4}, X_{1}, X_{2}, X_{3}\right), & N\left(0^{8}\right)=2 \\
0^{9}=\left(X_{4}, X_{1}, X_{3}, X_{2}\right), & N\left(0^{9}\right)=1 \\
0^{10}=\left(X_{2}, X_{4}, X_{3}, X_{1}\right), & N\left(0^{10}\right)=2 .
\end{array}
$$

The preference ordering $0 \triangleq\left\{0_{i j}\right\}$ appeared in the score sheet (12) is a fuzzy set. The score sheet (12) for the collective choice derives a $4 \times 4$ relation matrix $\left[\mu_{R}\right](13)$, whose elements are values of the membership function $\mu_{R}\left(X_{i}, X_{j}\right)$ for the fuzzy binary preference relation $R$ on $\left(X_{i}, X_{j}\right) \subseteq \tilde{X}_{i} \times \tilde{X}_{j}, i, j=1, \cdots, 4, i \neq j$

$$
\left[\mu_{R}\right]=\left(\begin{array}{llll}
0 & 0.75 & 0.75 & 0.7 \\
0.25 & 0 & 0.65 & 0.8 \\
0.25 & 0.35 & 0 & 0.6 \\
0.25 & 0.2 & 0.4 & 0
\end{array}\right) .
$$

2) Strength-of-Preference Rule:

$$
\mu_{R}\left(x_{i}, x_{j}\right)=\frac{1}{m} \sum_{k} w^{k} N^{k}\left(0_{i j}\right)=S\left(0_{i j}\right) / m
$$

where $N^{k}\left(0_{i j}\right)$ denotes a total score (e.g., number of vote) for a preference ordering $0_{i j}$ weighted by $w^{k} \in w, k=$ $1, \cdots, K$. The weighting coefficient $w^{k}$ represents a strength of preference for each ordered pair $\left(X_{i}, X_{j}\right)$ of attributes. 
The $m$ is a number of voters multiplied by a maximum value of $w^{k}$, viz. $m \triangleq \max \left(w^{k} n\right)$. For instance, suppose that $w \triangleq\left(w^{1}, w^{2}, w^{3}\right)=(3,2,1), \quad m=60$, and following scores for the binary relations (15) can be obtained from the original score sheet (12)

$$
\begin{array}{lll}
0_{12}=\left(X_{1}, X_{2}\right), & N\left(0_{12}\right)=15, & S\left(0_{12}\right)=38 \\
0_{13}=\left(X_{1}, X_{3}\right), & N\left(0_{13}\right)=15, & S\left(0_{13}\right)=37 \\
0_{14}=\left(X_{1}, X_{4}\right), & N\left(0_{14}\right)=14, & S\left(0_{14}\right)=35 \\
0_{21}=\left(X_{2}, X_{1}\right), & N\left(0_{21}\right)=5, & S\left(0_{21}\right)=13 \\
0_{23}=\left(X_{2}, X_{3}\right), & N\left(0_{23}\right)=13, & S\left(0_{23}\right)=32 \\
0_{24}=\left(X_{2}, X_{4}\right), & N\left(0_{24}\right)=16, & S\left(0_{24}\right)=42 \\
0_{31}=\left(X_{3}, X_{1}\right), & N\left(0_{31}\right)=5, & S\left(0_{31}\right)=12 \\
0_{32}=\left(X_{3}, X_{2}\right), & N\left(0_{32}\right)=7, & S\left(0_{32}\right)=16 \\
0_{34}=\left(X_{3}, X_{4}\right), & N\left(0_{34}\right)=12, & S\left(0_{34}\right)=28 \\
0_{41}=\left(X_{4}, X_{1}\right), & N\left(0_{41}\right)=5, & S\left(0_{41}\right)=10 \\
0_{42}=\left(X_{4}, X_{2}\right), & N\left(0_{42}\right)=4, & S\left(0_{42}\right)=8 \\
0_{43}=\left(X_{4}, X_{3}\right), & N\left(0_{43}\right)=8, & S\left(0_{43}\right)=21 .
\end{array}
$$

Using the score sheet (15), the relation matrix (16) can be derived

$$
\left[\mu_{R}\right]=\left(\begin{array}{llll}
0 & 0.63 & 0.62 & 0.58 \\
0.22 & 0 & 0.53 & 0.7 \\
0.2 & 0.26 & 0 & 0.47 \\
0.17 & 0.13 & 0.53 & 0
\end{array}\right)
$$

\section{B. Transitivity Checks}

Now, the problem is to find the nonfuzzy collective preference ordering from the fuzzy set (12) or (15). In general cases the relation matrix can represent connectivity, but intransitivity will occur in some triples $\left(X_{i}, X_{j}, X_{k}\right)$ $\in \tilde{X}_{i} \times \tilde{X}_{j} \times \tilde{X}_{k}$.

For example, in the relation matrix (13) under the simple majority rule, intransitivity occurs in triples $\left(X_{1}, X_{2}, X_{4}\right)$, $\left(X_{4}, X_{1}, X_{2}\right)$, and $\left(X_{4}, X_{3}, X_{2}\right)$. The relation matrix (16) under the strength-of-preference rule also includes intransitivity in triples $\left(X_{1}, X_{2}, X_{4}\right),\left(X_{3}, X_{2}, X_{1}\right),\left(X_{4}, X_{1}, X_{2}\right)$ and $\left(X_{4}, X_{3}, X_{2}\right)$.

Thus the problem is to ensure the transitivity for every binary preference relation. According to the definition of fuzzy transitivity (5), there are three ways to revise the original relation matrix: 1) to augment the value of $\left.\mu_{R}\left(X_{i}, X_{k}\right) ; 2\right)$ to lessen the value of $\mu_{R}\left(X_{i}, X_{j}\right) \wedge$ $\mu_{R}\left(X_{j}, X_{k}\right)$; and 3$)$ to lessen the value of $\mu_{R}\left(X_{i}, X_{j}\right)$ or the value of $\mu_{R}\left(X_{j}, X_{k}\right)$ discretionally. With those alternative devices, different revised relation matrices with the transitivity can be obtained. Transitivity checks can be performed sequentially in the revising processes. An effective computer program, ICOPSS/FR, is available for the transitivity checks to obtain the weak-ordered preference relations. The computer program can generate the transitive matrix internally. For example, the way 1) can derive the following revised matrix (17) with transitivity from the original intransitive relation matrix (13) under the majority rule

$$
\left[\mu_{R}^{\prime}\right]=\left(\begin{array}{llll}
0 & 0.75 & 0.75 & 0.75 \\
0.25 & 0 & 0.65 & 0.8 \\
0.25 & 0.35 & 0 & 0.6 \\
0.25 & 0.35 & 0.4 & 0
\end{array}\right)
$$

Fig. 1 shows the sequential procedure for revising the original matrix (13) with transitivity checks to obtain the revised matrix (17).

The way 2) can derive another revised matrix (18) with transitivity from the original intransitive relation matrix (13)

$$
\left[\mu_{R}^{\prime \prime}\right]=\left(\begin{array}{llll}
0 & 0.7 & 0.75 & 0.7 \\
0.25 & 0 & 0.65 & 0.8 \\
0.20 & 0.2 & 0 & 0.6 \\
0.2 & 0.2 & 0.4 & 0
\end{array}\right)
$$

The original relation matrix (16) under the strength-ofpreference rule can derive a revised matrix (19) assuring the transitivity with the way 1 )

$$
\left[\mu_{R}^{\prime}\right]=\left(\begin{array}{llll}
0 & 0.63 & 0.62 & 0.63 \\
0.22 & 0 & 0.53 & 0.7 \\
0.22 & 0.26 & 0 & 0.47 \\
0.22 & 0.26 & 0.35 & 0
\end{array}\right)
$$

The way 2) derives another revised matrix (20) from the original matrix (16)

$$
\left[\mu_{R}^{\prime \prime}\right]=\left(\begin{array}{llll}
0 & 0.58 & 0.62 & 0.58 \\
0.2 & 0 & 0.53 & 0.7 \\
0.13 & 0.13 & 0 & 0.47 \\
0.13 & 0.13 & 0.35 & 0
\end{array}\right)
$$

The process to obtain the weak-ordered matrix (20) with the computer assistance is shown in Fig. 2.

Finally the revised matrices which embody the transitivity are presented to the assessors as candidates for acceptance. In any way, elements appeared in the alternative matrices can be regarded as average weights for preferences revealed in the pairwise comparison in the collective choice under a selection rule. Thus, in practice, it can be asked if the changed values of the weighting parameters would be acceptable for the assessors. If the revised values in one of final matrices are more acceptable to the assessors, the nonfuzzy set representing a weak-ordered collective preference relation has been obtained.

\section{Finding an Acceptance Level of a Nonfuzzy Preference Ordering.}

Now an algorithm for deriving a nonfuzzy preference ordering among the attributes is discussed. The procedure is to decompose the fuzzy set of preference ordering like (12) into a union of the $\alpha$-level sets based on the finallyobtained transitive relation matrix. For instance, assume that the revised matrix (19) is finally accepted. Then the 


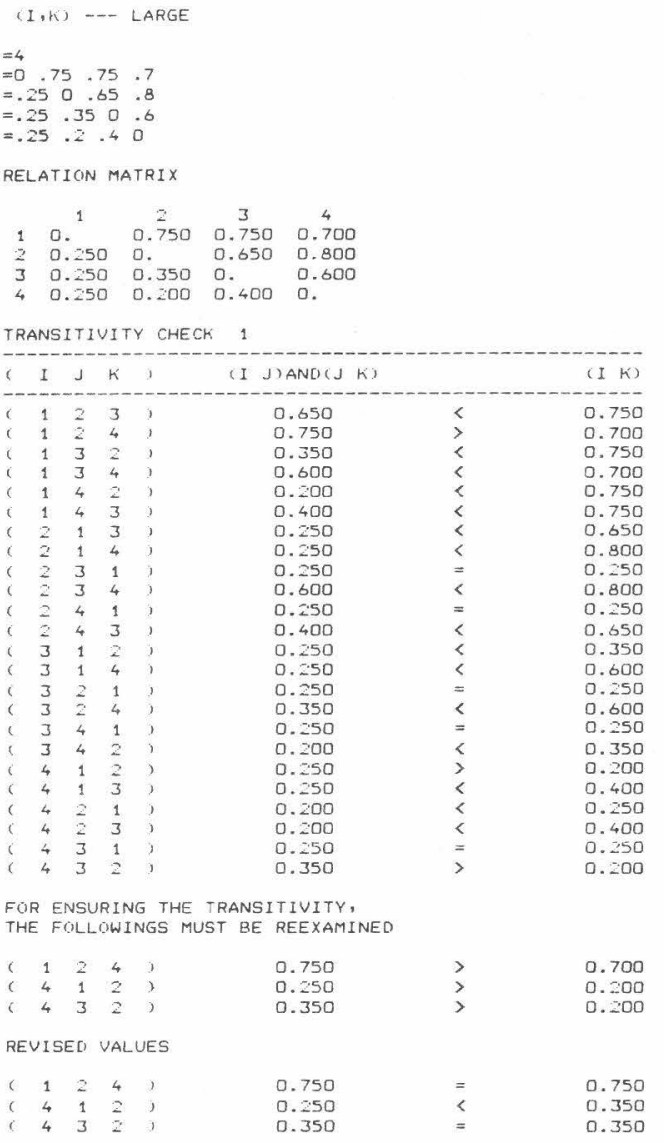

REVISEO RELATION MATRIX 1

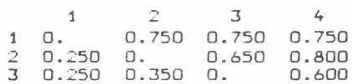

$4.050 \quad 0.350 \quad 0.4000$.

TRANSITIVITY CHECK 2

\begin{tabular}{|c|c|c|c|c|c|c|}
\hline C I & J & h & , & (I J)ANOCS K) & & $\left(\begin{array}{ll}1 \\
K\end{array}\right)$ \\
\hline 1 & 2 & 3 & , & 0.650 & $<$ & 0.750 \\
\hline$i$ & 2 & 4 & ) & 0.750 & $=$ & 0.750 \\
\hline 1 & 3 & 2 & ' & 0.350 & $<$ & 0.750 \\
\hline 1 & 3 & 4 & ) & 0.600 & $<$ & 0.750 \\
\hline 1 & 4 & 2 & ' & 0.350 & $<$ & 0.750 \\
\hline 1 & 4 & 3 & , & 0.400 & $<$ & 0.750 \\
\hline 2 & 1 & 3 & , & 0.250 & $<$ & 0.650 \\
\hline 12 & 1 & 4 & , & 0.250 & $<$ & 0.800 \\
\hline 2 & 3 & 1 & $y$ & 0.250 & $=$ & 0.250 \\
\hline 62 & 3 & 4 & , & 0.600 & $<$ & 0.800 \\
\hline 2 & 4 & 1 & 1 & 0.250 & $=$ & 0.250 \\
\hline 2 & 4 & 3 & 3 & 0.400 & $<$ & 0.650 \\
\hline 63 & 1 & 2 & 1 & 0.250 & $<$ & 0.350 \\
\hline 63 & 1 & 4 & ) & 0.250 & $<$ & 0.600 \\
\hline 63 & 2 & 1 & 3 & 0.250 & $=$ & 0.250 \\
\hline i 3 & 2 & 4 & ) & 0.350 & $<$ & 0.600 \\
\hline 63 & 4 & 1 & 3 & 0.250 & $=$ & 0.250 \\
\hline ( 3 & 4 & 2 & ) & 0.350 & $=$ & 0.350 \\
\hline 4 & 1 & 2 & , & 0.250 & $<$ & 0.350 \\
\hline ( 4 & 1 & 3 & $j$ & 0.250 & $<$ & 0.400 \\
\hline 64 & 2 & 1 & ) & 0.250 & $=$ & 0.250 \\
\hline 84 & $\overline{2}$ & 3 & 3 & 0.350 & $<$ & 0.400 \\
\hline 4 & 3 & 1 & ) & 0.250 & $=$ & 0.250 \\
\hline 4 & 3 & 2 & ) & 0.350 & $=$ & 0.350 \\
\hline
\end{tabular}

Fig. 1. Transitivity check with the way 1) (ICOPSS/FR) following $\alpha$-level decomposition can be obtained

$$
\begin{aligned}
& R_{\alpha=0.7}=\left(X_{2}, X_{4}\right) \\
& R_{\alpha=0.6}=R_{\alpha=0.7} \cup\left(\left(X_{1}, X_{2}\right),\left(X_{1}, X_{3}\right),\left(X_{1}, X_{4}\right)\right) \\
& R_{\alpha=0.5}=R_{\alpha=0.6} \cup\left(X_{2}, X_{3}\right) \\
& R_{\alpha=0.4}=R_{\alpha=0.5} \cup\left(X_{3}, X_{4}\right) \\
& R_{\alpha=0.3}=R_{\alpha=0.4} \cup\left(X_{4}, X_{3}\right) \\
& R_{\alpha=0.2}=R_{\alpha=0.3} \cup\left(\left(X_{2}, X_{1}\right),\left(X_{3}, X_{1}\right),\left(X_{3}, X_{2}\right),\right. \\
& \left.\left(X_{4}, X_{1}\right),\left(X_{4}, X_{2}\right)\right) .
\end{aligned}
$$

The classes of the nonfuzzy weak-ordered set is defined corresponding to the $\alpha$-level decomposition (21) as follows

$$
\begin{array}{ll}
C_{1}=\left(X_{2}, X_{4}\right) & \cdots \text { level } 0.7 \\
C_{2}=\left(\left(X_{1}, X_{2}\right),\left(X_{1}, X_{3}\right),\left(X_{1}, X_{4}\right)\right) & \cdots \text { level } 0.6 \\
C_{3}=\left(X_{2}, X_{3}\right), & \cdots \text { level } 0.5 \\
C_{4}=\left(X_{3}, X_{4}\right) & \cdots \text { level } 0.4 .
\end{array}
$$

Taking the intersection, we can find the collective ordering

$$
C_{1} \cap C_{2} \cap C_{3} \cap C_{4}=\left(X_{1}, X_{2}, X_{3}, X_{4}\right),
$$

or in another expression,

$$
X_{1} \succ X_{2} \succ X_{3} \succ X_{4} \text {. }
$$

Taking the minimum $\alpha$-level, we can call (24) the weakordered nonfuzzy relation in the level 0.4 for our decision problem. Or preferably, using the membership function $\mu_{C_{1} \cap C_{2} \cap C_{3} \cap C_{4}}\left(X_{i}, X_{j}\right)=0.47$, we can say about the collective preference ordering (24) "the degree or strength of agreement is 0.47 ." With this device, the range and degree of disagreement can be also ascertained. For instance, counterordering classes can be obtained from the $\alpha$-level set (21):

$$
\begin{array}{rlr}
C_{1}^{\prime}= & \left(X_{4}, X_{3}\right) \\
C_{2}^{\prime}= & \left(\left(X_{2}, X_{1}\right),\left(X_{3}, X_{1}\right),\left(X_{3}, X_{2}\right)\right. & \\
& \left.\left(X_{4}, X_{1}\right),\left(X_{4}, X_{2}\right)\right) . & \ldots \text { level } 0.3
\end{array}
$$

Taking the intersection, we can find a counterordering of (24)

$$
C_{1}^{\prime} \cap C_{2}^{\prime}=\left(X_{4}, X_{3}, X_{2}, X_{1}\right) .
$$

Taking the maximum $\alpha$-level, we can say about (24) "it is disagreeable in level 0.3." Or preferably, using the membership function $\mu_{C_{i}^{\prime}+C_{2}^{\prime}}\left(X_{i}, X_{i}\right)=0.35$, "the degree or strength of disagreement is 0.35 ." A transitivity closure representing the finally-obtained relation matrix can be depicted with a modified Hasse diagram (Fig. 3).

Clearly it is found that, in the case of the strength-ofpreference rule, the agreement level of the weak-ordered nonfuzzy relation (24) will be usually lowered in comparison to the simple majority case: like 0.6 to 0.4 (cf. Seo and Sakawa [15]). In other words, the strength-of-preference rule usually admits a higher degree of fuzziness in the agreement level for the collective choice. 
*RUN MAT-N

$(I, J) A N D(J, K)---$ LITTLE

$=4$

$=0.63 .62 \cdot .58$

$=.220 .53 .7$

$=.17^{\circ} \cdot 13 \cdot 350$

RELATION MATRIX

$\begin{array}{lcccc}1 & 2 & 3 & 4 \\ & 1 & 0.630 & 0.620 & 0.580\end{array}$

$\begin{array}{lllll}1 & 0 . & 0.630 & 0.620 & 0.580 \\ 2 & 0.220 & 0 . & 0.530 & 0.700\end{array}$

$\begin{array}{lllll}3 & 0.220 & 0 . & 0.530 & 0.700 \\ 4 & 0.200 & 0.260 & 0 . & 0.470\end{array}$

$\begin{array}{lllll}4 & 0.170 & 0.130 & 0.350 & 0.4\end{array}$

TRANSITIVITY CHECK 1

\begin{tabular}{|c|c|c|c|c|c|c|}
\hline $6 \quad 1$ & $J$ & k & ) & J) AND (J & & (I K) \\
\hline 1 & 2 & 3 & ) & 0.530 & $<$ & 0.620 \\
\hline < 1 & 2 & 4 & ) & 0.630 & $>$ & 0.580 \\
\hline 6 & 3 & 2 & 2 & 0.260 & $<$ & 0.630 \\
\hline 6 & 3 & 4 & ) & 0.470 & $<$ & 0.580 \\
\hline$c$ & 4 & 2 & ) & 0.130 & $<$ & 0.630 \\
\hline 8 & 4 & 3 & ) & 0.350 & $<$ & 0.620 \\
\hline ( 2 & 1 & 3 & ) & 0.220 & $<$ & 0.530 \\
\hline 62 & 1 & 4 & ) & 0.220 & $<$ & 0.700 \\
\hline 6 & 3 & 1 & , & 0.200 & $<$ & 0.220 \\
\hline 6 & 3 & 4 & ) & 0.470 & $<$ & 0.700 \\
\hline$c$ & 4 & 1 & ) & 0.170 & $<$ & 0.220 \\
\hline 6 & 4 & 3 & ) & 0.350 & $<$ & 0.530 \\
\hline$c$ & 1 & 2 & ) & 0.200 & $<$ & 0.260 \\
\hline$c$ & 1 & 4 & ) & 0.200 & $<$ & 0.470 \\
\hline c & 2 & 1 & ) & 0.220 & $>$ & 0.200 \\
\hline 3 & 2 & 4 & ) & 0.260 & $<$ & 0.470 \\
\hline 6 & 4 & 1 & ) & 0.170 & $<$ & 0.200 \\
\hline ( 3 & 4 & 2 & ) & 0.130 & $<$ & 0.260 \\
\hline c & 1 & 2 & ) & 0.170 & $>$ & 0.130 \\
\hline 6 & 1 & 3 & ) & 0.170 & $<$ & 0.350 \\
\hline 4 & 2 & 1 & ) & 0.130 & $<$ & 0.170 \\
\hline 4 & 2 & 3 & ) & 0.130 & $<$ & 0.350 \\
\hline 4 & 3 & 1 & ) & 0.200 & $>$ & 0.170 \\
\hline 4 & 3 & 2 & ) & 0.260 & $>$ & 0.130 \\
\hline
\end{tabular}

FOR ENSURING THE TRANSITIVITY,

THE FOLLOWINGS MUST BE REEXAMINED

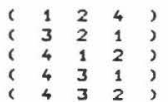

$$
\begin{aligned}
& 0.630 \\
& 0.220 \\
& 0.170 \\
& 0.200
\end{aligned}
$$

0.200

$>$
$>$
$>$
$>$
$>$

0.580
0.200

0.200

0.170
0.130

REVISED VALUES

$\left(\begin{array}{llll}6 & 2 & 4\end{array}\right.$

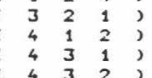

0.580

0. 130

0.130
0.170

0.130

REVISED RELATION MATRIX,

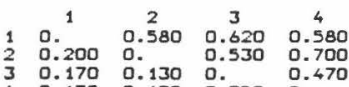

\begin{tabular}{|c|c|c|c|c|c|c|c|}
\hline 6 & 1 & $J$ & k & ) & (I J)ANDCS & & (I K) \\
\hline 6 & 1 & 2 & 3 & ) & 0.530 & $<$ & 0.620 \\
\hline s & 1 & 2 & 4 & ) & 0.580 & $=$ & 0.580 \\
\hline 6 & 1 & 3 & 2 & ) & 0.130 & $<$ & 0.580 \\
\hline 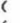 & 1 & 3 & 4 & ) & 0.470 & $<$ & 0.580 \\
\hline 6 & 1 & 4 & 2 & ) & 0.130 & $<$ & 0.580 \\
\hline 6 & 1 & 4 & 3 & ) & 0.350 & $<$ & 0.620 \\
\hline 6 & 2 & 1 & 3 & ) & 0.200 & $<$ & 0.530 \\
\hline 6 & 2 & 1 & 4 & ) & 0.200 & $<$ & 0.700 \\
\hline 6 & 2 & 3 & 1 & , & 0.170 & $<$ & 0.200 \\
\hline 6 & 2 & 3 & 4 & ) & 0.470 & $<$ & 0.700 \\
\hline 6 & 2 & 4 & 1 & ) & 0.130 & $<$ & 0.200 \\
\hline 6 & 2 & 4 & 3 & ) & 0.350 & $<$ & 0.530 \\
\hline 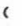 & 3 & 1 & 2 & ) & 0.170 & $>$ & 0.130 \\
\hline 6 & 3 & 1 & 4 & ) & 0.170 & $<$ & 0.470 \\
\hline ， & 3 & 2 & 1 & ) & 0.130 & $<$ & 0.170 \\
\hline c & 3 & 2 & 4 & ) & 0.130 & $<$ & 0.470 \\
\hline c & 3 & 4 & 1 & ) & 0.130 & $<$ & 0.170 \\
\hline$c$ & 3 & 4 & 2 & ) & 0.130 & $=$ & 0.130 \\
\hline$c$ & 4 & 1 & 2 & ) & 0.130 & $=$ & 0.130 \\
\hline$c$ & 4 & 1 & 3 & ' & 0.130 & $<$ & 0.350 \\
\hline ( & 4 & 2 & 1 & ? & 0.130 & $=$ & 0.130 \\
\hline$c$ & 4 & 2 & 3 & 1 & 0.130 & $<$ & 0.350 \\
\hline 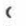 & 4 & 3 & 1 & , & 0.170 & $>$ & 0.130 \\
\hline 6 & 4 & 3 & 2 & ) & 0.130 & $=$ & 0.130 \\
\hline \multicolumn{8}{|c|}{$\begin{array}{l}\text { FOR ENSURING THE TRANSITIVITY, } \\
\text { THE FOLLOWINGS MUST BE REEXAMINED }\end{array}$} \\
\hline 6 & 3 & 1 & 2 & ) & 0.170 & $>$ & 0.130 \\
\hline 6 & 4 & 3 & 1 & , & 0.170 & $>$ & 0.130 \\
\hline \multicolumn{8}{|c|}{ REVISED VALUES } \\
\hline 6 & 3 & 1 & 2 & ) & 0.130 & $=$ & 0.130 \\
\hline 6 & 4 & 3 & 1 & , & 0.130 & $=$ & 0.130 \\
\hline
\end{tabular}

TRANSITIVITY CHECK

REVISEO RELATION MATRIX

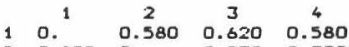

$\begin{array}{lllll}2 & 0.200 & 0 . & 0.530 & 0.700\end{array}$

$\begin{array}{lllll}3 & 0.130 & 0.130 & 0 . & 0.470 \\ 4 & 0.130 & 0.130 & 0.350 & 0 .\end{array}$

\begin{tabular}{|c|c|c|c|c|c|c|c|c|}
\hline$c$ & I & $J$ & k & ) & (I & J)AND( $J$ K) & & (I K) \\
\hline 6 & 1 & 2 & 3 & ) & & 0.530 & $<$ & 0.620 \\
\hline s & 2 & 2 & 4 & , & & 0.580 & $=$ & 0.580 \\
\hline 6 & 1 & 3 & 2 & , & & 0.130 & $<$ & 0.580 \\
\hline$c$ & 1 & 3 & 4 & ) & & 0.470 & $<$ & 0.580 \\
\hline$c$ & 1 & 4 & 2 & ) & & 0.130 & $<$ & 0.580 \\
\hline 6 & 1 & 4 & 3 & , & & 0.350 & $<$ & 0.620 \\
\hline 6 & 2 & 1 & 3 & ) & & 0.200 & $<$ & 0.530 \\
\hline 6 & 2 & 1 & 4 & ) & & 0.200 & $<$ & 0.700 \\
\hline$c$ & 2 & 3 & 1 & ) & & 0.130 & $<$ & 0.200 \\
\hline 6 & 2 & 3 & 4 & ) & & 0.470 & $<$ & 0.700 \\
\hline$c$ & 2 & 4 & 1 & ) & & 0.130 & $<$ & $0.2 \mathrm{C}-1+3$ \\
\hline i & 2 & 4 & 3 & ) & & 0.350 & $<$ & 0.53 \\
\hline$c$ & 3 & 1 & 2 & ) & & 0.130 & $=$ & 0.130 \\
\hline$c$ & 3 & 1 & 4 & ' & & 0.130 & $<$ & 0.47 \\
\hline$c$ & 3 & 2 & 1 & ) & & o. 130 & $=$ & o. 130 \\
\hline c & 3 & 2 & 4 & ) & & 0.130 & $<$ & 0.470 \\
\hline 6 & 3 & 4 & 1 & ) & & 0.130 & $=$ & 0.130 \\
\hline c & 3 & 4 & 2 & ) & & 0.130 & $=$ & 0.130 \\
\hline 6 & 4 & 1 & 2 & ) & & 0.130 & $=$ & 0.13 \\
\hline 6 & 4 & 1 & 3 & , & & 0.130 & $<$ & 0.350 \\
\hline 6 & 4 & 2 & 1 & ) & & 0.130 & $=$ & 0.130 \\
\hline 6 & 4 & 2 & 3 & ) & & 0.130 & $<$ & 0.350 \\
\hline c & 4 & 3 & 1 & ) & & 0.130 & $=$ & 0.130 \\
\hline 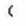 & 4 & 3 & 2 & ) & & 0.130 & $=$ & 0.130 \\
\hline
\end{tabular}

TRANSITIVITY CHECK 3

Fig. 2. Transitivity check with the way 2) (ICOPSS/FR)

\section{CONSTRUCtion of the Fuzzy Multiattribute UTILITY FUNCTION}

Now the second phase of the fuzzification process in step 2) should be examined.

The fuzzy multiattribute utility function (FMUF) can be assessed on the numerical values of the scaling constants $k_{i}, i=1, \cdots, m$, whose numerical ordering has been determined beforehand corresponding to the nonfuzzy prefer- ence ordering for the attributes $X_{i}, i=1, \cdots, m$. Even though the indifference experiment among the attributes $X$ has been executed as if it is a single-agent decision problem, construction of the collective preference ordering in the prior stage will have mitigated to some extent arbitrariness in the magnitude of the scaling constants.

After the indifference experiment, the FMUF can be derived with fuzzy scaling constants $\tilde{k}_{i}, i=1, \cdots, m$, treated as fuzzy numbers such as the $L-R$ type. The $L-R$ 


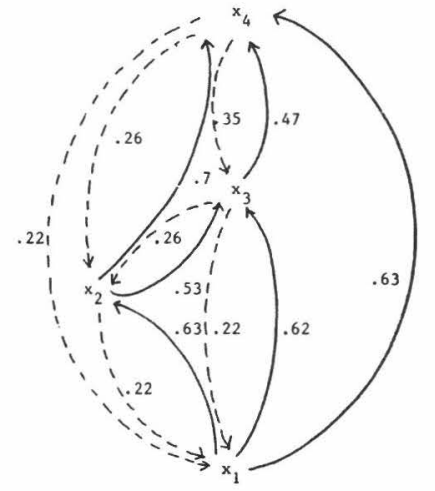

Fig. 3. A transitivity check for a collective choice.

type fuzzy number is defined as follows (Dubois and Prade [5]).

Definition 2 ( $L-R$ Type Fuzzy Number): A $L-R$ type fuzzy number $\tilde{n}$ is expressed in the following form

$$
\tilde{n} \triangleq(\bar{n}, \alpha, \beta)
$$

where $\bar{n}$ is a "mean" value, and $\alpha$ and $\beta$ show left- and right-side dispersion. A membership function of the fuzzy number $\tilde{n}$ (27) is

$$
\mu_{\tilde{n}}= \begin{cases}L((\bar{n}-z) / \alpha), & z \leqslant \bar{n}, \alpha>0 \\ R((z-\bar{n}) / \beta), & \bar{n} \leqslant z, \beta>0\end{cases}
$$

where $L(0)=R(0)=1$. After obtaining the relative values $k_{i} / k_{s}$ of the scaling constants in (1) and (2) with the indifference experiment, a numerical value of $k_{s}$ for the utility function $u_{s}\left(x_{s}\right)$ of the most preferable (regarded) attribute should be determined. For this purpose, the lottery experiment on the $m$ attributes is executed answering the following question:

Question: Let us consider the lottery that will take alternatively with a probability $p_{s}$ the best values $x_{i 1}$ for all attributes $X_{i}, i=1, \cdots, m$, and with a probability $1-p_{s}$ the worst values $x_{i 0}$ for all the attributes. On the other hand, let us consider the certain consequence in which the most preferable attribute takes its best value $x_{s 1}$, and all other attributes take their worst values $\left\{x_{\bar{s} 0}\right\}, \bar{s}=1, \cdots, s$ $-1, s+1, \cdots, m$. Then what is the numerical value of the probability $p_{s}$ with which the lottery and the certain consequence will become indifferent?

The fuzzification device here is to take the probability $p_{s}$ as a fuzzy number $\tilde{p}_{s} \triangleq\left(\bar{p}_{s}, \beta, \gamma\right)$, where $\bar{p}_{s}$ denotes a "mean" value of $\tilde{p}_{s}$, and $\beta$ and $\gamma$ denote respectively the left and right side spreads from $\bar{p}_{s}$. The parameters $\beta, \gamma$, and $\bar{p}_{s}$ can be obtained as minimum, maximum, and medium valuations of the probability $p_{s}$ in the collective choice or the group decisionmaking. Then the scaling constant $k_{s}$ can be assessed by

$$
\tilde{p}_{s}=\tilde{k}_{s} \triangleq\left(\bar{k}_{s}, \beta, \gamma\right)
$$

from the representation theorem 1 .
We can use the L-R type (28) for fuzzy number $\tilde{k}_{s}$ and perform the algebraic operations on it. The corresponding utility value $u_{s}$ in (1) and (2) can be also reexamined as a fuzzy number $\tilde{u}_{s} \triangleq\left(\bar{u}_{s}, \delta, \gamma\right)$, where the parameters $\delta, \gamma$, and $\bar{u}_{s}$ can be obtained respectively as minimum, maximum and medium values of reevaluations in the group decisionmaking. With those fuzzy numbers $\tilde{k}_{s}$ and $\tilde{u}_{s}$, all the fuzzy scaling constants $\tilde{k}_{i}, i=1, \cdots, m, i \neq s$, of the multiattribute utility functions (1), (2) can be obtained from the representation theorem 1 .

The fuzzification of the scaling constants will have some effects on choosing the representation forms (multiplicative or additive) of the MUFs. For this reason, the following representation check should be performed

a) if $\sum_{i=1}^{m} \tilde{k}_{i}>1$, then $-1<K<0$ (multiplicative)

b) if $\sum_{i=1}^{m} \tilde{k}_{i}<1$, then $K>0$ (multiplicative)

c) if $\sum_{i=1}^{m} \tilde{k}_{i}=1$, then $K=0$ (additive).

Because the scaling constant $\hat{k}_{i}, i=1, \cdots, m$, is a fuzzy number, the assertion ( $>$ and $<$ ) for that is still fuzzy. Thus it should be asked what the truth value of the assertion " $\sum_{i=1}^{m} \tilde{k}_{i}$ is greater (or smaller) than 1 " is. The separation theorem for two fuzzy sets (Zadeh [16], Dubois and Prade [6]) can be applied to the comparison of the fuzzy numbers $\sum_{i}^{m} \tilde{k}_{i}$ and $\tilde{1}=(\overline{1}, 0,0)$. The separation theorem asserts that when $M$ is the maximum grade of the intersection of two-bounded convex fuzzy sets, the degree of separation $D$ of those sets is obtained by $D=1-M$. For making an answer to the question, we can choose a threshold level $\theta, 1>\theta>0$. If $M>\theta$ then it can be asserted that $\sum_{i=1}^{m} \tilde{k}_{i}=\tilde{1}$ in the level $\theta$ (Fig. 4).

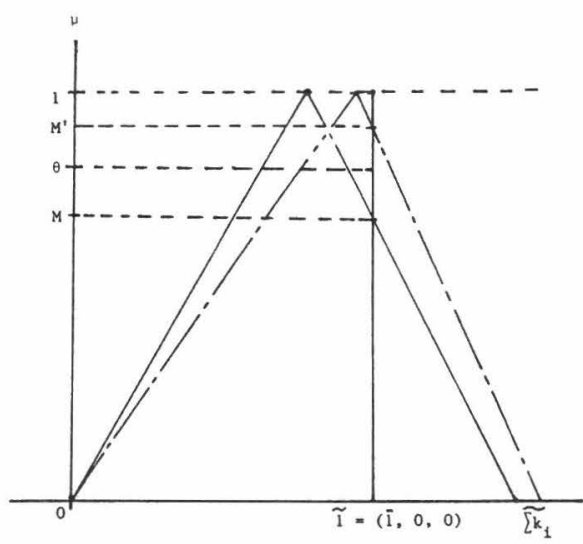

Fig. 4. Representation check with the separation theorem. 
In the result, the FMUF can be derived as a fuzzy number in more sophisticated forms:

additive

$$
\tilde{U}\left(x_{1}, \cdots, x_{m}\right)=\sum_{i=1}^{m} \tilde{k}_{i} \tilde{u}_{i}\left(x_{i}\right)
$$

or multiplicative

$$
1+K \tilde{U}\left(x_{1}, \cdots, x_{m}\right)=\sum_{i=1}^{m}\left(K \tilde{k}_{i} \tilde{u}_{i}\left(x_{i}\right)+1\right) .
$$

The values of FMUF are calculated for alternative policies in which different numerical values of the attributes $x_{i}, i=1, \cdots, m$, are assigned. The values of the singleattribute utility function $u_{i}\left(x_{i}\right), i=1, \cdots, m, i \neq s$ can be also assessed as the fuzzy number $\tilde{u}_{i}=\left(\bar{u}_{i}, 0,0\right)$ or $\hat{u}_{i}=$ $\left(\bar{u}_{i}, \xi, \nu\right)$ where $\bar{u}_{i}, \xi$ and $\nu$ can be determined from the reevaluation by the collective decisionmaking as stated before. Based on the assessment of FMUF for the alternative policies $A, B, C, \cdots$, a preference ordering can be found. For instance,

$$
\tilde{U}(C)>\tilde{U}(B)>\tilde{U}(A)>\cdots \Leftrightarrow C>B>A \cdots .
$$

Because the comparison (33) on the fuzzy number $\tilde{U}$ is still fuzzy, the previous procedure for ascertaining if $\tilde{U}(C)$ is truly larger than $\tilde{U}(B)$, etc. should be applied here again. Thus the priority of the best preferred policy $C$, for example, can be confirmed with a threshold level.
Thus fuzzification of the multiattribute utility functions (MUF) can be primarily considered in terms of diversified evaluation in the context of collective choice or multipleagents decision problems.

\section{Summary and Concluding Remarks}

The operational procedure for deriving the FMUF described in this paper is summarized in Fig. 5.

The main problem of collective choice is to resolve the interpersonal value-conflicts by articulating value trade-offs among multiple decisionmakers. However, due to the difficulty of interpersonal comparison of individual preferences on a common unit of measurement, a device for pretreatment of conflict resolution should be developed, which will narrow down a range of the conflicts embeded in the diversification of evaluations. In this sense, fuzzy extension of MUF method can be a promising direction for manipulating the collective choice or group decision problems. In addition, with this device the annoying aggregation problem to compose the "social" utility function based on individual preference orderings can be avoided.

A computer program ICOPSS/FR, based on ICOPSS/I (Sakawa and Seo [13]), has been developed for deriving a nonfuzzy weak-ordered set in the collective choice. This program can be used for finding the relation matrix that embodies the transitivity in some agreement level. In addition, ICOPSS/FR can execute the fuzzy number operations rapidly (Figs. 6 and 7). This facility will raise

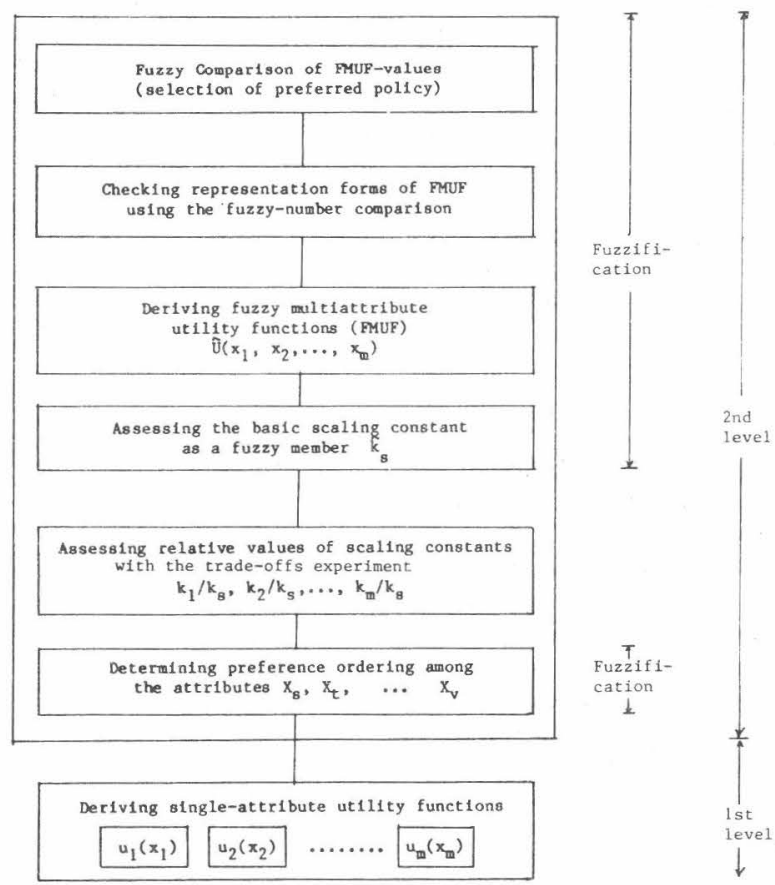

Fig. 5. Operational method for deriving the FMUF. 


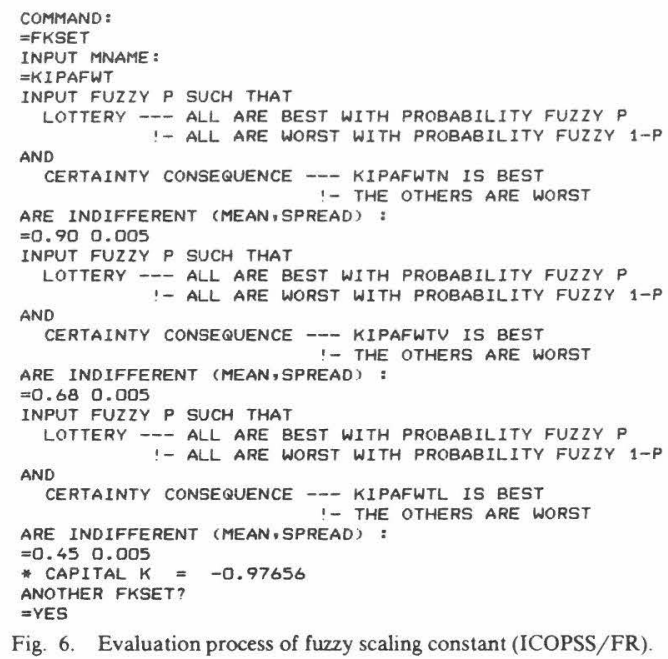

Fig. 6. Evaluation process of fuzzy scaling constant (ICOPSS/FR).

applicability and feasibility of multiattribute utility analysis in the collective choice problems.

\section{REFERENCES}

[1] K. J. Arrow, "A difficulty in the concept of social welfare," $J$. Political Economy, vol, 58, pp. 328-346, 1950.

[2] K. J. Arrow, Social Choice and Individual Values. New York Wiley, 1951

[3] J. M. Blin, "Fuzzy relations in group decision theory," J. Cybernetics, vol. 4, no. 2, pp. 17-22, 1974.

[4] J. M. Blin and A. B. Whinston, "Fuzzy sets and social choice," $J$ Cybernetics, vol. 3, no 4, pp. 28-36, 1974

[5] D. Dubois and H. Prade, "Operations on fuzzy numbers," Int. J. Syst. Sci., vol. 9, no. 6, pp. 613-626, 1978.

[6] "Systems of linear fuzzy constraints," Fuzzy Sets and Systems, vol. 3, pp. 37-48, 1980.

[7] J. S. Dyer and R. K. Sarlin, "Group preference aggregation rules based on strength of preference," Management Sci., vol. 25, no. 9 , pp. 822-832, 1979.

[8] R. L. Keeney, "Multiplicative utility functions, Oper. Res., vol. 22, pp. 22-34, 1974.

[9] , "A group preference axiomatization with cardinal utility," Management Sci., vol. 23, no. 2, pp. 140-145, 1976.

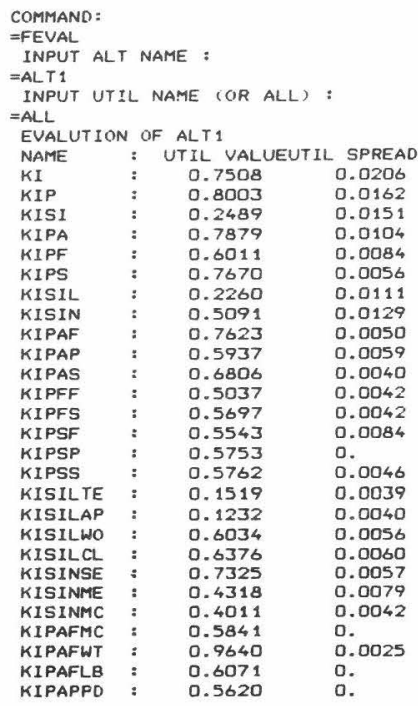

Fig. 7. Final evaluation of FMUF (ICOPSS/FR).

[10] R. L. Keeney and C. W. Kirkwood, "Group decision making using cardinal social welfare functions," Management Sci., vol. 22, no. 4 . 1975.

[11] R. L. Keeney and H. Raiffa, Decisions with Multiple Objectives, Preferences and Value Tradeoffs. New York: Wiley, 1976.

[12] M. Sakawa and F. Seo, "Integrated methodology for computer-aided decision analysis," in Progress in Cybernetics and Systems Research, T. Robert, F. de P. Hanika and R. Tomlinson, Eds. Washington: Hemisphere, 1982.

[13] "An interactive computer program for subjective systems and its application," IIASA, WP-80-64, 1980

[14] F. Seo and M. Sakawa, "An experimental method for diversified evaluation and risk assessment with conflicting objectives," IEEE Trans. Syst. Man, Cybern., vol. 14, no. 2, pp 213-223, 1984.

[15] "Fuzzy assessment of multiattribute utility functions, in Interactive Decision Analysis and Interpretative Computer Intelligence, A. Wierzbicki and M. Grauer, Eds. New York: SpringerVerlag, 1984, pp. 97-104.

[16] L. A. Zadeh, "Fuzzy sets," Inform. and Contr., vol. 8, pp. 338-353, 1965.

[17] , "Similarity relations and fuzzy orderings," Inform. Sci., vol. 3, pp. 177-200, 1971. 
